www.nature.com/clinicalpractice/neuro

administration ceased. The authors conclude that $A \beta^{\star} 56$ induces memory impairment through transient physiological, rather than permanent neuropathological, brain alterations.

Lesné et al. propose that the targeting of soluble $A \beta$ memory-disrupting assemblies in humans could be a potential mechanism for halting Alzheimer's disease before permanent structural damage is incurred. Their results suggest that other soluble protein assemblies could also have a role in inducing brain dysfunction.

Pippa Murdie

Original article Lesné S et al. (2006) A specific amyloid- $\beta$ protein assembly in the brain impairs memory. Nature 440: 352-357

\section{Intensive patient education improves clinical outcome in migraine patients}

Migraine patients, it has been reported, are among those most dissatisfied with their standard of medical care. A wide range of therapies for migraine are now available, but demands on clinician time make it difficult to educate patients to derive maximum benefit from these treatments. Rothrock et al. sought to determine whether the addition of an intensive patient education program to routine patient management might improve the clinical outcome for migraine patients. The researchers randomly allocated 100 consecutive patients to receive either routine care (controls) or a standardized course of instruction on migraine biogenesis and management. The course of instruction involved three 90 min classes taught by migraine sufferers who had previously undergone intensive instruction by a neurologist.

At 6 months, the intervention group had a significantly greater reduction in mean MIGRAINE DISABILITY ASSESSMENT (MIDAS) score than did the control group. There was a threefold greater frequency of prescription drug use and more analgesic overuse in the control group compared with the intervention group, and there were four times as many headache-related calls to a doctor and twice as many unscheduled visits for acute headache medication by patients in the control group.

The authors state that their education program, costing US\$5,000/year, improved clinical outcome and patient compliance, and reduced demand for medical resources. They conclude that traditional management is inadequate to meet the needs of migraine patients and call for further initiatives to improve patient care.

Jim Casey

Original article Rothrock JF et al. (2006) The impact of intensive patient education on clinical outcome in a clinic-based migraine population. Headache [doi: 10.1111/j.1526-4610.2006.00428.x]

\section{Glucose mediates cognitive dysfunction in type 2 diabetes}

Older adults with type 2 diabetes are known to be at an increased risk for cognitive dysfunction. It is unknown if this is related to chronically elevated glucose levels or insulin levels; however, the available evidence suggests that elevated insulin levels might be to blame. A US team has compared the effects of rosiglitazone (which increases insulin sensitivity) and glibenclamide (which encourages insulin production, and is also known as glyburide) on cognition in older patients with type 2 diabetes.

In total, 145 patients (mean age 60 years) were randomly assigned to 24 weeks of daily therapy with either rosiglitazone or glibenclamide. Doses were titrated throughout the study to achieve equal glycemic control for all patients (range 4-8mg for rosiglitazone and 2.5-15 mg for glibenclamide). The patients completed seven cognitive tests at baseline and again at week 24 . The tests fell into three categories: working memory, learning ability, and cognitive efficiency.

Performance changed significantly only on one of the three tests that assessed working memory, the CANTAB PAL TEST. Both rosiglitazone and glibenclamide were associated with similar improvements in patient performance from baseline $(P<0.0001$ and $P<0.001$, respectively). No relationship was observed between circulating insulin levels and performance; however, a fall in fasting plasma glucose was correlated with improvement, regardless of treatment group.

Although the mechanism is currently unknown, high peripheral glucose levels are thought to adversely affect cerebral glucose, which has been shown to be important in animal studies of working memory. These results indicate that glucose, rather than insulin, mediates cognitive dysfunction in diabetic adults.

Katherine Sole

Original article Ryan CM et al. (2006) Improving metabolic control leads to better working memory in adults with type 2 diabetes. Diabetes Care 29: 345-351

\section{GLOSSARY}

MIGRAINE DISABILITY ASSESSMENT (MIDAS)

A questionnaire that

measures headache-related disability by counting the number of days of lost and limited activity due to migraine

CANTAB PAL TEST

The Cambridge

Neuropsychological Test Automated Battery Paired Associates Learning test; it assesses memory and new learning 